Mon. Not. R. Astron. Soc. 000,1-?? (2010) Printed 15 November $2018 \quad$ (MN LATEX style file v2.2)

\title{
Positron Annihilation on Polycyclic Aromatic Hydrocarbon molecules in the Interstellar Medium
}

\author{
N. Guessoum ${ }^{1 \star}$, P. Jean ${ }^{2}$, and W. Gillard ${ }^{3}$ \\ ${ }^{1}$ American University of Sharjah, Physics Department, PO Box 26666, Sharjah, UAE \\ ${ }^{2}$ CESR, CNRS/Université de Toulouse, B.P. 4346, 31028 Toulouse Cedex 4, France \\ ${ }^{3}$ KTH, Department of Physics, AlbaNova University Centre, SE-10691 Stockholm, Sweden
}

Accepted 2009 October 27. Received 2009 October 27; in original form 2009 September 6

\begin{abstract}
We examine the annihilation of positrons on polycyclic aromatic hydrocarbon (PAH) molecules in interstellar medium conditions. We estimate the annihilation rates of positrons on PAHs by a semi-empirical approach. We show that PAHs can play a significant role in the overall galactic positron annihilation picture and use the annihilation rates and INTEGRAL galactic emission measurements to constrain the amount of PAHs present in the ISM. We find an upper limit of $4.6 \times 10^{-7}$ for the PAH abundance (by number, relative to hydrogen).
\end{abstract}

Key words: ISM: abundances - ISM: molecules - gamma-rays: theory.

\section{INTRODUCTION: POSITRON ISM ANNIHILATION ISSUES AND INTERROGATIONS}

In Astrophysics, the origin of positrons annihilating in the interstellar medium (ISM) and the nature and distribution of PAH molecules in space represent two unsolved problems which do not, at least on the surface, appear to have anything to do with one another.

The abundant presence of positrons in our galaxy, especially in its bulge, and the high rate of annihilation determined through its gamma-ray line signature at $511 \mathrm{keV}$ have been established for a few decades now Leventhal. MacCallum, \& Stang 1978; Purcell et al. 1997; Harris et al. 1998; Milne et al. 2000; Jean et al. 2003; Knödlseder et al. 2005), after the initial observation of the radiation by Johnson. Harnden, \& Havmes (1972), Johnson, \& Havmes (1973), and Havmes et al. (1975). The flux and profile of the annihilation line, and thus the rate of steady production and annihilation of positrons have now been measured rather precisely by several instruments, balloon-borne and satellite-carried detectors (see, most recently, Harris et al. 1998; Knödlseder et al. 2005; Weidenspointner et al. 2008). The analysis of the data on the gamma-ray emission (line and continuum) resulting from the annihilation of positrons on gas in various ISM regions has allowed for a better understanding of the processes and conditions of annihilation (Churazov et al. 2005; Jean et al. 2006). Still, the origin of positrons has re-

* E-mail: $\quad$ nguessoum@aus.edu, jean@cesr.fr, gillard@particle.kth.se mained mostly a mystery, for the simple reason that no single astronomical population of objects is known to have both the spatial distribution that the annihilation radiation map presents and the capability to produce such high rates of positrons (for a brief review of the problem, see Guessoum, Jean, \& Prantzos 2006).

The story of the PAH molecules and their role in the ISM parallels that of the positrons. A few decades ago, the radiation emission signatures of these molecules, namely lines in the Infra-Red spectrum of dark nebulae, seemed to point to their abundant presence in the Galaxy (Gillett, Forrest, \& Merrill 1973 and others later). A large effort by researchers (Dulev \& Williams 1981; Leger \& Puget 1984; Allamandola. Tielens, \& Barker 1985) and others later) helped identify characteristics of these molecules, including their structure, energy levels, and charge states; this was supposed to help pinpoint the presence and distribution of specific $\mathrm{PAH}$ molecules in different ISM regions. The latter goal has yet to be reached (see for instance Ruiterkamp et al. 2005; Cami et al. 2005), so that even though it is now widely believed that roughly $10 \%$ perhaps more - of the carbon in the ISM can be found in PAH molecules (Tielens 1990), definitely identifying any of them has remained an elusive goal.

The connection between positrons and PAH molecules in earthly laboratories was made about a decade ago when experimental positron groups started to measure positron-molecule annihilation cross sections and realized that these increase by several orders of magnitudes when molecules are large (Surko et al. 1988; Iwata et al. 1995; Iwata, Greaves, \& Surko 1996; Barnes, Gilbert, \& Surko 2003). A substantial amount of experimental and theoretical 
work has been performed on this topic, and measurements of cross sections for a small dozen PAH and alkane molecules bombarded by positrons have been published, leading to theoretical efforts to explain the strong "Feshbach vibrational" resonance displayed in such interactions (see the extensive review of Surko, Gribakin, \& Buckman 2005). A few researchers hinted at the possible importance of such reactions in the ISM, but no serious consideration of this issue has been attempted heretofore.

The closest that astrophysicists came to addressing this issue was when the interaction of positrons with dust in the ISM was treated in detail Guessoum, Jean, \& Gillard 2005). In that work, dust was considered in the three forms it is usually presented in: "big grains", "very small grains", and PAHs. And because the positron-dust-grain cross section was assumed to be essentially geometric, "very small grains" were neglected next to "big grains", which themselves proved to be relevant only in the hot phases of the ISM and, in some special conditions, in the warm ionized phase. It seemed reasonable then to conclude that PAHs, being even smaller and much less abundant, would play a negligible role in the positron's life and death in the ISM. But that was not correct, since the positron-PAH cross section was not geometrical but rather highly resonant, especially at very low energies/temperatures; indeed, with cross sections sometimes a million times larger than those of charge exchange with hydrogen atoms, abundances of PAHs of about $10^{-6}$ (by number, $\mathrm{N}_{\mathrm{PAH}} / \mathrm{N}_{\mathrm{H}}$ ) would make them roughly as important as other species.

We must stress, however, that PAHs, although commonly considered as the "molecular end" of the dust grain size distribution, behave very differently with respect to positrons. That is why the treatment of positron annihilation on PAHs is warranted now and is fundamentally distinct from all past (astrophysical) works.

In this work, we investigate the extent to which $\mathrm{PAH}$ molecules are relevant in the positrons' annihilation in the ISM. We have thus assembled the relevant information regarding PAHs in the ISM (abundances, sizes, distributions, charge states, etc.), and on the interactions between positrons and PAHs. With that we estimate the rate of positron annihilation on PAH molecules in the various ISM phases and compare it to those of other processes Guessoum, Jean, \& Gillard 2005).

In the next section we briefly review current knowledge about PAH molecules in the ISM. In section 3 we present the main experimental information on positron annihilation on PAHs and then proceed to determine the positron-PAH reaction rate at $8000 \mathrm{~K}$ (i.e. in the warm media) by several steps. In section 4 we use that knowledge to calculate the rate of annihilation of positrons on PAHs in the relevant phases of the ISM, paying particular attention to the $\mathrm{PAH}$ charging effect. In section 5 we explore the observational consequences of our calculations using the measurements performed with the spectrometre SPI on the space observatory INTEGRAL (INTErnational Gamma-Ray Astrophysics Laboratory). In section 6 we summarize our findings and present our main conclusions regarding the possible importance of this process in current positron astrophysical studies and point to the work that will be needed in the future in order to advance our understanding of this problem.

\section{PAHS IN ASTROPHYSICS}

Polycyclic Aromatic Hydrocarbons are organic molecules which consist entirely of $\mathrm{C}$ and $\mathrm{H}$ atoms and have a polycyclic structure, which makes the energy needed for breaking them up much higher than average; this then helps them survive in space. In addition, each ring has 6 electrons from the carbon atoms "floating" around and contributing to the binding. These molecules have "aromatic" properties. The prototype example of a $\mathrm{PAH}$ is Naphthalene $\left(\mathrm{C}_{10} \mathrm{H}_{8}\right)$, which is made of two rings. A simpler example would be benzene $\left(\mathrm{C}_{6} \mathrm{H}_{6}\right)$, but it consists of only one ring, so it is not "polycyclic".

These molecules became the focus of important astrophysical research when the "unidentified infrared (UIR) emission bands" (3-13 $\mu \mathrm{m})$ that had been observed from nebulae since the early seventies were shown (Leger \& Puget 1984; Allamandola. Tielens, \& Barker 1985), more than a decade later, to be very similar to those produced by PAHs when they are temporarily heated (in space by UV radiation); UIR bands then came to be referred to as AIBs (aromatic IR bands). Indeed, the usual dust grain populations could not be responsible for such emissions because they were too big and thus could not be heated to 1000 $\mathrm{K}$ or more and cooled quickly. Using observations made by space-borne IR telescopes, similar IR emission bands were later found in a variety of objects, ranging from comets to galaxies (e.g. Ehrenfreund et al. 2002). Salama et al. (1999) concluded that "PAHs are ubiquitous throughout the general diffuse ISM". Such identifications allowed for an inference of the abundance of PAHs in the ISM, roughly believed to be $10^{-7}-10^{-6}$ (by number, i.e. $\mathrm{N}_{\mathrm{PAH}} / \mathrm{N}_{\mathrm{H}}$ ) Allain 1996), making them the most abundant molecules in the ISM after $\mathrm{H}_{2}$ and $\mathrm{CO}$.

PAHs are often seen as an extension of, or even the seeds for, the small dust grain populations (Allain 1996; Salama et al. 1999; Peeters 2002; Abergel et al. 2005). Indeed, not only are they the largest molecules known in space, but the largest of them have been described (Peeters 2002; Rapacioli 2005) as aggregates of planar molecules, which when stacked can become the "very small grains" of dust, which range in size from $1 \mathrm{~nm}$ to $10 \mathrm{~nm}$. In fact, it is postulated that PAHs constitute the first step in the formation of dust grains.

The above-mentioned emission lines are generally associated with $\mathrm{C}-\mathrm{C}$ and $\mathrm{C}-\mathrm{H}$ vibrational modes of PAHs of various sizes (Dulev \& Williams 1981 first, and many researchers subsequently). It is also often concluded that PAHs with a rather large number of carbons (greater than about 30) are responsible for some of the AIB features, particularly those around $6.2 \mu \mathrm{m}$ and $11.3 \mu \mathrm{m}$. Since the photodissociation threshold energy is a few tenths of $\mathrm{eV}$ per carbon atom, it is clear that "small" molecules (with a number of carbon atoms $\mathrm{N}_{C} \lesssim 30$ ) would more easily be depleted in H II regions by energetic photons Allain 1996; Peeters 2002; Abergel et al. 2005); bigger ones easily survive. It has also been postulated that $\mathrm{UV}$, cosmic rays, and shock waves could chemically alter such molecules, particular in warm/hot, collisionally active environments. Finally, PAHs can be neutral or electrically charged, depending on the densities of the photoionizing radiation and of free electrons (Omont 1986; Draine \& Sutin 1987; Lepp et al. 1988; 
Table 1. Measured $Z_{e f f}$ for PAH molecules at room temperature (Iwata et al. 1996; Iwata et al. 1997).

\begin{tabular}{llc}
\hline Molecule & Chemical Formula & $Z_{\text {eff }}$ \\
\hline \hline Benzene & $\mathrm{C}_{6} \mathrm{H}_{6}$ & 18000 \\
Toluene & $\mathrm{C}_{7} \mathrm{H}_{8}$ & 190000 \\
Naphtalene & $\mathrm{C}_{10} \mathrm{H}_{8}$ & 494000 \\
Anthracene & $\mathrm{C}_{14} \mathrm{H}_{10}$ & 4330000 \\
\hline
\end{tabular}

Bakes \& Tielens 1994; Allain 1996; Dartois \& d'Hendecourt 1997; Weingartner \& Draine 2001a b; Abergel et al. 2005), and their IR emission spectra are then significantly different.

\section{CURRENT LABORATORY DATA ON POSITRON ANNIHILATION ON PAHS}

As mentioned in the introduction, interest in $\mathrm{e}^{+}-\mathrm{PAH}$ annihilation grew with the development of experimental techniques that allowed for the measurement of cross sections of positrons annihilating with more and more complex atoms and molecules (Iwata, Greaves, \& Surko 1996, 1997; Iwata et al. 2000; Surko et al. 2000; Gilbert et al. 2000; Sullivan et al. 2002; Barnes, Gilbert, \& Surko 2003; Marler et al. 2004; Surko, Gribakin, \& Buckman 2005; Surko 2006). Indeed, it soon became apparent that $\mathrm{e}^{+}-\mathrm{PAH}$ reactions exhibited huge cross sections, or equivalently reaction rates or $Z_{e f f}$, the latter being defined as:

$Z_{e f f}=\frac{\lambda}{\pi r_{0}^{2} c}$

where $\lambda$ is the annihilation rate (in $\mathrm{cm}^{3} / \mathrm{s}$ ), and $r_{0}$ is the classical electron radius; it turns out that $Z_{\text {eff }}$ has essentially nothing to do with the number of electrons $\mathrm{Z}$ of the species (for example, $Z_{\text {eff }}=8$ for $\mathrm{H}$ ), and indeed reaches values of $10^{7}$ or more for $\mathrm{PAH}$ molecules (see the references given above). However, only relatively small PAH molecules have been experimentally investigated so far (Iwata, Greaves, \& Surko 1996, 1997; Barnes, Gilbert. \& Surko 2003), as molecules with more than 3 aromatic rings have such low vapor pressures that performing those measurements is a great experimental challenge. The only PAHs for which a $Z_{\text {eff }}$ was actually experimentally measured (Iwata, Greaves, \& Surko 1996, 1997) are listed in Table 1. In the Iwata. Greaves, \& Surko (1996) experiment the FWHM of the annihilation line resulting from positrons on Naphthalene was also measured and found to be $2.29 \mathrm{keV}$ (compared to $1.71 \mathrm{keV}$ for $\mathrm{H}_{2}$ ).

The very large values for $Z_{\text {eff }}$ have been theoretically interpreted in terms of resonant interaction between the positron and the molecule, or equivalently by the formation of temporary positron-molecule bound states. Gribakin (2000) has come up with a theoretical model to reproduce the experimental data on the cross sectional profiles in terms of the positron momentum distribution and of the temperature of the medium; he also produced a simple fit for the exponential increase of $Z_{\text {eff }}$ in terms of the number of atoms in the aromatic molecules (he found $Z_{\text {eff }} \propto N^{8.2}$, where $\mathrm{N}$ is the total number of atoms in the molecule). We note, however, that $Z_{\text {eff }} / Z$ tends to saturate for larger N's in other types of molecules (see, for instance, the data on alkanes in Fig. 8 of Barnes, Gilbert, \& Surko $(2003))$. None of the studies which investigated the variations of $Z_{\text {eff }}$ with the physical characteristics of molecules could satisfactorily reproduce the saturation effect for large molecules (Murphy \& Surko 1991; Laricchia \& Wilkin 1997, 1998; Iwata et al. 2000). This saturation is clearer when values of $Z_{e f f} / Z$ are presented as a function of the number of electrons $Z$ of the molecules (see Iwata et al. 1995 and Fig. 39 of Surko, Gribakin, \& Buckman 2005) and we thus propose a different fit. We have found the relation :

$\ln \left(\frac{Z_{\text {eff }}}{Z}\right)=A\left(1-e^{-\frac{Z}{B}}\right)$

to give a satisfactory fit (see Fig. 1); the number of measurements is much greater for alkanes than for PAHs; we show that $Z_{\text {eff }}$ 's for the two types of molecules converge in this representation. In our fitting function (Eq. 2), the best parameter values are: $A=10.75 \pm 0.44, B=40.1 \pm 3.8$. We hasten to add that this relation was, like Gribakin's, not based on any physical considerations, only the fact that experiments show that $Z_{\text {eff }} / Z$ levels off for large molecules. We should add that $\mathrm{e}^{-}-\mathrm{PAH}$ cross sections, being non-resonant, are not of much help in this regard; like Gribakin, our only recourse was an empirical fit.

We must emphasize that the experimental values of $Z_{\text {eff }}$ were obtained at room temperature only, whereas the temperature we are mainly interested in is $8000 \mathrm{~K}$, since positron annihilation in the ISM is believed to occur mostly in the warm (neutral and ionized) phases, where $\mathrm{T} \approx 8000 \mathrm{~K}$ Churazov et al. 2005; Jean et al. 2006) and PAHs are expected to be easily evaporated in the hot phase (where $\mathrm{T}$ $\sim 10^{6} \mathrm{~K}$ ). To our knowledge, only one experiment has measured $Z_{\text {eff }}$ as a function of $\mathrm{T}$ (Iwata et al. 2000), but this was limited to very few and small molecules (methane $\mathrm{CH}_{4}$, ethylene $\mathrm{C}_{2} \mathrm{H}_{4}$, and butane $\mathrm{C}_{4} \mathrm{H}_{10}$ ) and to temperatures up to about $2500 \mathrm{~K}$. More importantly, we note that measurement errors are largely unknown (rarely, if ever, given in the scant literature); consequently, the uncertainties we have given above (for $A$ and $B$ ) are related to the fit only, and the few measurements that are available do not allow us to constrain the "saturation" of the $Z_{\text {eff }}$ function (in terms of Z) very well. We do insist, however, on the existence of such a saturation, unlike what the Gribakin function implies, and that in turn has important consequences on the relative role of PAH's in the annihilation of positrons in the ISM.

So we need to determine the value of $Z_{\text {eff }}$ at $8000 \mathrm{~K}$, for which we need measurements of the cross section as a function of the positron energy. Some experiments (see Barnes, Gilbert, \& Surko 2003 and Surko, Gribakin. \& Buckman 2005) have performed such measurements, so we can simply calculate $Z_{\text {eff }}$ as a function of the positron temperature by way of the reaction rate $\lambda$ :

$\lambda=\langle\sigma v\rangle=\int_{0}^{\infty} \frac{2}{\sqrt{\pi}} \frac{\sqrt{E}}{(k T)^{3 / 2}} e^{-E / k T} \sigma(E) v d E$,

where $\sigma$ is the annihilation cross section, v the positron velocity, and $\mathrm{E}$ its kinetic energy.

Noting that at low temperatures $Z_{\text {eff }}$ drops as $T^{-1 / 2}$ (see Figure 8 of Iwata et al. 2000), the above approach would yield the right behavior for $Z_{\text {eff }}$ only if the cross sections 


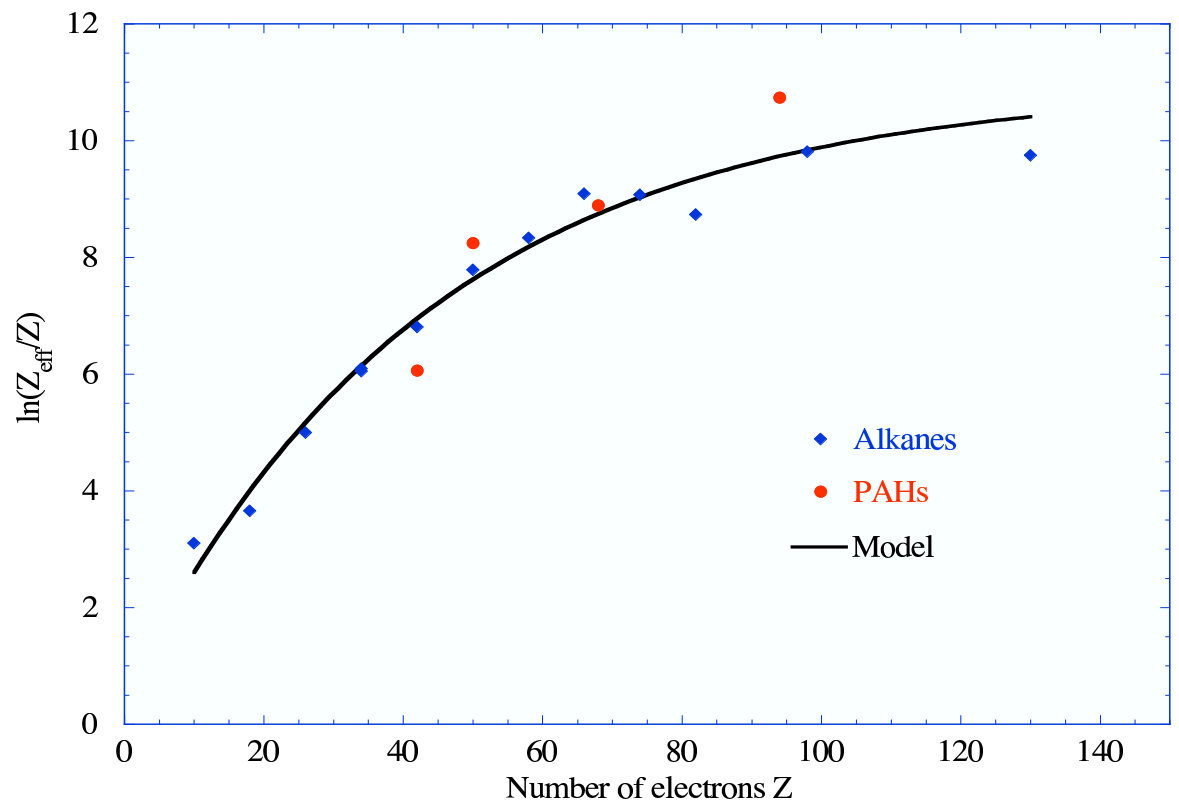

Figure 1. Comparison of $\ln \left(Z_{e f f} / Z\right)$ for alkanes and PAHs as functions of the number of electrons in the molecule Z (Iwata et al. 1995). Also shown is the best fit of the empirical function (see Eq. 1) obtained with $A=10.75$ and $B=40.1$.

Table 2. Calculated $Z_{\text {eff }}$ at $\mathrm{T}=8000 \mathrm{~K}$ for a few (mediumlarge) Alkane (top group) and PAH molecules (bottom group).

\begin{tabular}{llc}
\hline Molecule & Chemical Formula & $Z_{\text {eff }}$ \\
\hline \hline Butane & $\mathrm{C}_{4} \mathrm{H}_{10}$ & $6.4 \times 10^{3}$ \\
Hexane & $\mathrm{C}_{6} \mathrm{H}_{14}$ & $5.5 \times 10^{4}$ \\
Heptane & $\mathrm{C}_{7} \mathrm{H}_{16}$ & $1.8 \times 10^{5}$ \\
Octane & $\mathrm{C}_{8} \mathrm{H}_{18}$ & $3.5 \times 10^{5}$ \\
Nonane & $\mathrm{C}_{9} \mathrm{H}_{20}$ & $6.8 \times 10^{5}$ \\
n-Dodecane & $\mathrm{C}_{12} \mathrm{H}_{26}$ & $2.6 \times 10^{6}$ \\
\hline Naphthalene & $\mathrm{C}_{10} \mathrm{H}_{8}$ & $4.2 \times 10^{5}$ \\
Anthracene & $\mathrm{C}_{14} \mathrm{H}_{10}$ & $4.1 \times 10^{6}$ \\
Hexacene & $\mathrm{C}_{26} \mathrm{H}_{16}$ & $2.4 \times 10^{7}$ \\
Octacene & $\mathrm{C}_{34} \mathrm{H}_{20}$ & $1.1 \times 10^{8}$ \\
Decacene & $\mathrm{C}_{42} \mathrm{H}_{24}$ & $1.5 \times 10^{8}$ \\
\hline
\end{tabular}

were extended at very low energies as $E^{-1}$. We were thus able to reproduce the experimental data of $Z_{e f f}(T)$ for butane rather well for most temperature ranges, although $Z_{\text {eff }}$ tends to decline very slowly at higher temperatures and not flatten out completely. Figures 2 ( $\mathrm{a}$ and $\mathrm{b}$ ) show the behavior of $Z_{\text {eff }}$ as a function of temperature for various molecules; Figure $2 \mathrm{~b}$ shows the variation of normalized values of $Z_{\text {eff }}$ on a linear scale so as to emphasize the weak dependence of $Z_{\text {eff }}$ on $T$.

Having noted the variation of $Z_{\text {eff }}$ with temperature for alkanes, which we assume to be the same for PAHs, we then produce values of $Z_{\text {eff }}$ at $8000 \mathrm{~K}$ for a few PAHs using Figure 1 and the T-dependence. In Table 2 we give values of $Z_{\text {eff }}$ for some alkane and PAH molecules.

We emphasize the large uncertainties inherent in these calculations due to the fact that only a few actual measurements exist for positron annihilation reactions with alkanes and/or small PAHs at low temperatures. We call for new experiments and extensive measurements as well as theoret- ical calculations of annihilation cross sections for positrons with as many PAH species as possible.

Barnes, Gilbert, \& Surko (2003) measured the rate of annihilation of low energy positrons with many hydrocarbon molecules. They found that $Z_{\text {eff }}$ is enhanced at positron energies that correspond to resonance energies of the strongest infrared active vibrational modes. They interpreted these results as excitations of vibrational Feshbach resonances by incoming positrons which are temporarily trapped on the molecules. Based on this observation, it would in principle be possible to infer the variation of the annihilation rate on PAHs as a function of the temperature of thermalized positrons by integrating the product of their (Maxwellian) velocity distribution with the spectrum of the vibrational modes of the PAH molecules. However, the observed resonance effect does not seem to be systematic. Indeed, there is no clear enhancement of $Z_{\text {eff }}$ for some energies of positrons (e.g. see Figs. 12 and 13 of Barnes, Gilbert, \& Surko 2003). Moreover, a shift in the position of the resonance with respect to the vibrational mode is observed, which seems to be linked to the binding energy of the positron to the molecule.

Referring to recent work on energy-resolved positron-molecule annihilation cross sections (Barnes, Young, \& Surko 2006), Surko has remarked (private communication) that the temperature dependence of $Z_{\text {eff }}$ referred to above $\left(Z_{\text {eff }} \propto T^{-1 / 2}\right.$ obtained by Iwata et al. 2000 up to $2500 \mathrm{~K}$ ) may not hold at 8000 $\mathrm{K}$, since the $1 / E$ variation of the cross section, which leads to the $T^{-1 / 2}$ dependence of $Z_{e f f}$, seems to apply only at very low energies $\left(E<<E_{r e s}\right)$, that is for direct annihilation, whereas for "high" temperatures, resonant effects change that behavior. Should $Z_{\text {eff }}$ decrease faster with $T$, the values at $8000 \mathrm{~K}$ would be significantly smaller than inferred here. However, this uncertain behavior is itself unclear, as we do not know the binding energies for the larger PAHs and thus the energy difference between those and the vibrational energy values. This shows that 

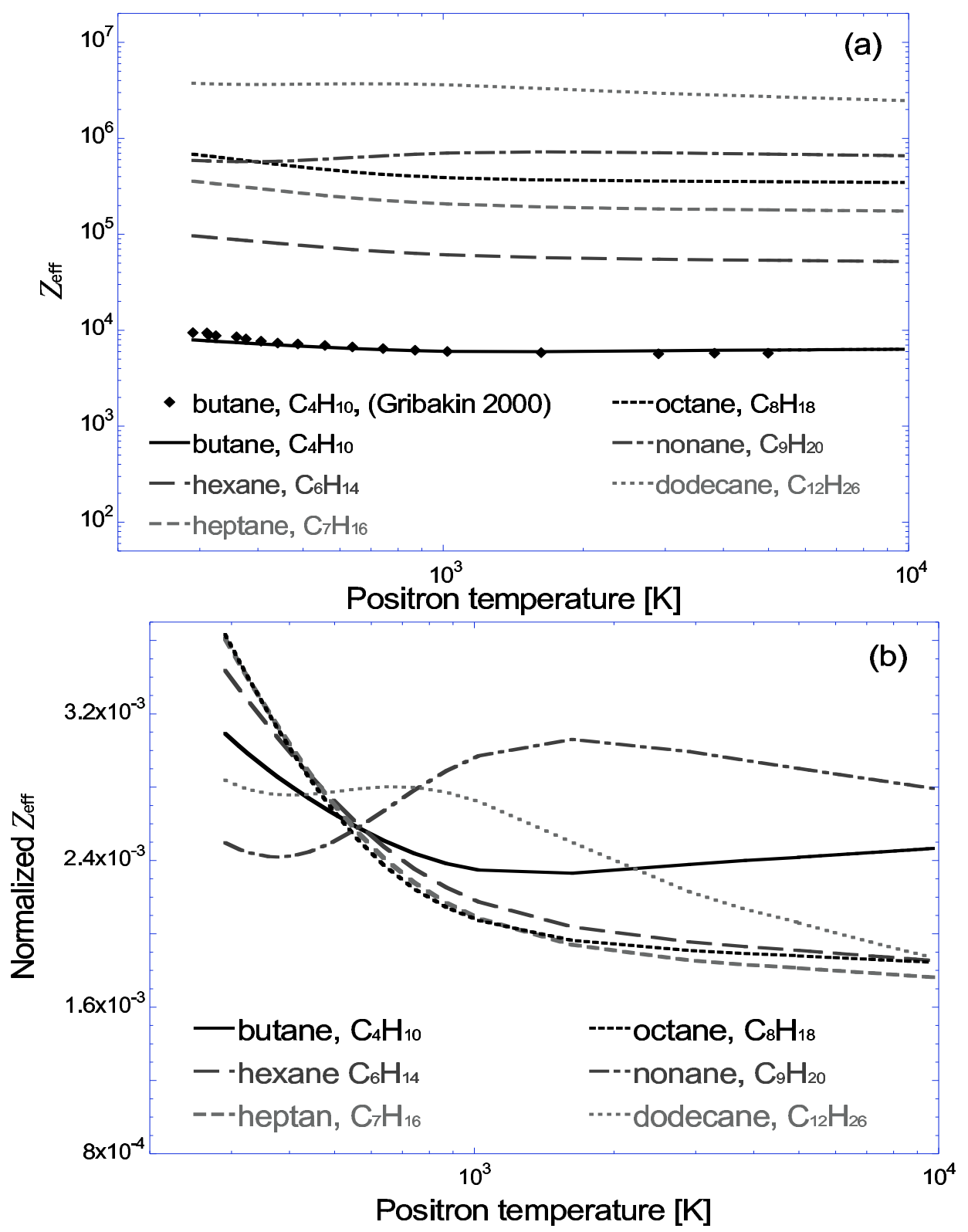

Figure 2. $Z_{\text {eff }}$ as a function of temperature for various molecules (alkanes and PAHs) in (a) logarithmic scale and (b) normalized to the value 1 .

additional (unknown) factors need to be taken into account in order to build a correct model.

Consequently, we cannot yet analytically obtain $Z_{\text {eff }}$ as a function of the temperature and of $\mathrm{N}$ (the number of atoms) in large PAHs with our current level of understanding of the annihilation of positrons with molecules. We are then left with the necessity of producing a numerical fit of the available data for $Z_{\text {eff }}(\mathrm{N})$ and then extrapolating for larger molecules, since it turns out that the prime contributors for positron annihilation on PAHs in the ISM are those with a number of atoms around 50. (See below the discussion about PAH size distributions and the contribution of each species to the annihilation rate.)

Since the larger PAH molecules will be the more relevant ones for our astrophysical positron considerations, we believe that this issue of $Z_{\text {eff }}$ for PAHs with $N_{C} \gtrsim 30$
$(N \gtrsim 50)$ is an important one that should be attempted experimentally at the earliest.

\section{ANNIHILATION OF GALACTIC POSITRONS ON PAHS (RELATIVE GALACTIC IMPORTANCE)}

We now set out to determine the rate of positron annihilation on PAH molecules in the ISM and compare it with those of positrons with the usual gas components (free electrons, atomic and molecular hydrogen, helium, dust grains). We may start from the reaction rate formula $\lambda=Z_{\text {eff }} \pi r_{0}^{2} c$ defining $Z_{\text {eff }}$, and sum up the contributions of the various PAH species through their number-abundances $y_{s}$ (the subscript s referring to any given species), considering that some 
may be partially destroyed, depending on the physical conditions in the medium. One must also take into account the possible electric charge of the molecule, which would then enhance or reduce the "affinity" between the positrons and the molecules.

The reaction rate for each $\mathrm{PAH}$ species can then be written as:

$\lambda_{e^{+-s}}=y_{s} Z_{e f f, s} \pi r_{0}^{2} c f_{\text {elec }-s}$,

and this would then be summed over for the various $\mathrm{PAH}$ species. Note that $y_{s}$ includes the destruction effects; that is, $y_{s}=y_{s 0} \times f_{\text {dest }-s}$, where $y_{s 0}$ represents the abundance of an s-species of PAH in the absence of any destruction effects; $f_{\text {dest-s }}$ and $f_{\text {elec-s }}$ will be estimated in the next sub-section.

\subsection{PAH charging and destruction:}

Several studies have been performed on the determination of the charge states of dust grains and PAH molecules of various sizes in different conditions and by several physical processes. Draine \& Sutin (1987) considered collisional effects and showed that charge states other than $0,+1$, and -1 have extremely low probabilities $\left(\sim 10^{-4}\right.$ or less $)$ in various physical conditions. This confirmed statements by Omont (1986). Lepp et al. (1988) considered diffuse ISM clouds and determined charge states in various regions of the cloud (edge, center, etc.); they concluded that the neutral state is most prevalent. Bakes \& Tielens (1994) then added photoelectric effects to the analysis and calculated the $f(Z)$ probability of finding a grain/PAH molecule in a charge state Z. Using typical densities, temperatures, and UV field intensities in various phases of the ISM, they found (their figure 6): charge states of mostly 0 and 1 (average of 0.5 ) and -1 and 0 (average of $\approx-0.25$ ) for the Warm and Cold ISM media, respectively. The work of these authors was revisited by Dartois \& d'Hendecourt (1997), who applied their ionization model to various conditions of the ISM and of nebulas; they found that in the diffuse ISM, $65 \%$ of large PAH molecules are negatively charged and $35 \%$ are neutral (different results are obtained for different physical conditions). Photoelectric charging was revisited by Weingartner \& Draine (2001a) with improved atomic data, grain size distributions, etc.; they found (their figure 9) that except for very large PAH molecules in the warm medium, where the average charge was $\approx 0.4$, all PAH's, especially mid-size molecules, will be neutral in practically all conditions.

As to how the charge state of a PAH molecule will affect the reaction rate of capture and annihilation of a positron, we refer to the thorough discussion of the physics and chemistry of interstellar PAHs by Omont (1986). This author tells us that because the charge of a PAH is essentially distributed over its entire surface, the processes which govern the charges of interstellar PAH's are very similar to those involved in the charging of interstellar dust grains. He thus concludes that one may reasonably adapt the classical discussions of the charging of interstellar grains to PAH's, basing this conclusion on several works (see references therein).

The positron-charged-PAH effect on the annihilation rate can then be dealt with in the same way as we did in Guessoum, Jean, \& Gillard (2005), i.e. as did Zurek (1985) and Guessoum. Ramaty, \& Lingenfelter (1991). That is, the reaction rate gets multiplied by an "electric charge factor", $\mathrm{f}_{\text {elec }}$, where $\mathrm{f}_{\text {elec }}$ is given by $\left(1-Z e^{2} / a_{s} k T\right)$ or $\exp \left(-Z e^{2} / a_{s} k T\right)$ depending on whether the molecule, with a radius $a_{s}$, is negatively or positively charged, and where $f_{\text {elec }}$ then takes the values of 3.1 and 0.12 in the $\mathrm{Z}=-1 /+1$ charge state, for a typical PAH size of $1 \mathrm{~nm}$.

As to the PAH destruction effect, we simply consider that in the hot phase of the ISM, electrons are more than energetic enough $(k T \sim 100 \mathrm{eV})$ to break the molecule every time a collision occurs, so that the PAHs are largely depleted from such an environment; however, in the warm and cold phases ( $T \approx 8000 \mathrm{~K}$ and $10-100 \mathrm{~K}$ respectively), collisions will rarely, if ever, destroy the PAH molecules; therefore $f_{\text {dest }}$ $=0$ for the hot phase and $f_{\text {dest }}=1$ for the others.

\section{2 $\mathrm{e}^{+}-\mathrm{PAH}$ reaction rate in the ISM:}

Considering that the PAH number-abundances of $10^{-7}-$ $10^{-6}$ quoted above are for all PAH molecules in the ISM, and noting (from Figure 1) that only large molecules will be effective annihilators of positrons, we must convolve the $Z_{\text {eff }}$ values shown in Figure 1 with the PAH size or atom-number distribution; Désert, Boulanger, \& Puget (1990) and Draine \& Lazarian (1998) give somewhat different PAH distributions, which Pilleri et al. (2009) present (their Figure 5) as a function of the number of carbon atoms in the molecule. The rates obtained using the two distributions differ by only $0.9 \%$; in the rest of this work, we adopt the more recent, "lognormal" distribution of Draine \& Lazarian (1998).

Putting all factors together then gives the following expression for the reaction rate between positrons and PAHs:

$\lambda_{e^{+}-P A H}=\left[\int_{N} Z_{\text {eff }}(N) d y_{s}(N) f_{\text {elec-s }}\right] \pi c r_{0}^{2} Y_{P A H}$,

where $Y_{P A H}$ refers to the total PAH abundance (relative to $\mathrm{H})$ in the ISM. The summation over species then gives:

$\lambda_{e^{+}-P A H} \approx 1.5 \times 10^{-13} \frac{Y_{P A H}}{10^{-6}}\left\langle f_{\text {elec }}\right\rangle \mathrm{cm}^{3} / \mathrm{s}$.

We should note, however, that taking into account the uncertainty on the semi-empirical model of $Z_{\text {eff }}$ (see section 3 ) the relative uncertainty on the derived rate is about $47 \%$.

We now compare this result with the rates for the main positron processes in the various ISM phases (charge exchange with $\mathrm{H}$, direct annihilation with free and bound electrons, radiative combination with electrons, capture by dust grains) as given in Guessoum. Jean, \& Gillard (2005); the values for all rates are given in Table 3 . We first note that if the total PAH abundance in the ISM is of the order of $10^{-7}$ or less, then positrons will not be affected by PAH molecules, however large they may be. If, however, $Y_{P A H} \sim 10^{-6}$, then the rate of annihilation of positrons by PAHs becomes non-negligible, especially if the molecules are neutral or negatively charged. Indeed, in the warm neutral phase this process becomes second in importance, with a rate up to $26 \%$ that of charge exchange with hydrogen when PAHs are negatively charged. Similarly, in the warm ionized phase the process is then second in importance, with a rate up to $39 \%$ that of radiation combination with free electrons. We must stress, however, that it is generally believed that PAHs would normally exist in multiple charge 
Table 3. Reaction rates for positron annihilation (by various processes) in the warm neutral (WNM) and ionized (WIM) phases of the ISM. The reaction rate for positron annihilation with PAHs is calculated using the total PAH number-abundance $Y_{P A H}=10^{-6}$

\begin{tabular}{lcc}
\hline Process & $\begin{array}{c}r_{e^{+}-s}\left(\mathrm{~cm}^{3} / \mathrm{s}\right) \\
\text { WNM }\end{array}$ & $\begin{array}{c}r_{e^{+}-s}\left(\mathrm{~cm}^{3} / \mathrm{s}\right) \\
\text { WIM }\end{array}$ \\
\hline \hline Charge exchange with H & $1.8 \times 10^{-12}$ & - \\
Direct annihilation with free electrons & - & $1.7 \times 10^{-13}$ \\
Direct annihilation with bound electrons & $4.4 \times 10^{-14}$ & -- \\
Radiative combination with electrons & - & $1.2 \times 10^{-12}$ \\
Capture by dust grains & $6.5 \times 10^{-15}$ & $4.6 \times 10^{-14}$ \\
Annihilation with PAH molecules & $1.5 \times 10^{-13}$ & $1.5 \times 10^{-13}$ \\
\hline
\end{tabular}

states, with perhaps a predominance of neutral molecules thus reducing their importance as annihilators.

\section{OBSERVATIONAL CONSEQUENCES}

One observational consequence of our calculation of the rate of positron annihilation on PAHs is the possible enhanced effect these molecules would have on the $511 \mathrm{keV}$ line produced in a nebula where these molecules may be abundant, especially if physical conditions (e.g. high collision rates) lead to a significant negative charging of the PAHs. And should PAH molecules, in such situations, start to dominate in annihilating positrons, the observed $511 \mathrm{keV}$ spectra would be different from the usual "general ISM" ones. Indeed, since measurements of the spectra of the annihilation of positrons with PAH molecules Iwata. Greaves, \& Surko 1997) have determined FWHM's of the annihilation line that are between 2.0 and $3.0 \mathrm{keV}$, the spectra from PAH-rich and negatively charged nebulae would be substantially wider than one recorded from a warm region of the ISM, where a line of width $1.5 \mathrm{keV}$ is produced. Furthermore, the positronium fraction (or $3 \gamma / 2 \gamma$ ratio) would likely be different in the two measurements.

$$
\text { Using the method presented in }
$$
Guessoum, Jean, \& Gillard (2005) we have calculated the spectral distribution of the annihilation emission including the contribution of PAHs with an abundance of $Y_{P A H} \sim 10^{-6}$, taking their electric charge into account. The FWHM of the annihilation line in PAH was here taken to be $\approx 2.5 \mathrm{keV}$. In the warm neutral medium, the positronium fraction does not change significantly: from $99.9 \%$ in the absence of PAHs to $99.4 \%$ and $98.6 \%$ with neutral and negatively charged PAHs, respectively. In the warm neutral medium, the shape of the $511 \mathrm{keV}$ line is not notably modified. The situation is quite different in the warm ionized medium. The positronium fraction decreases from $87 \%$ without PAHs to $78 \%$ and $64 \%$ with neutral and negatively charged PAHs, respectively. The influence of PAHs distinctly deforms the base of the spectral line (see Fig. 4).

Such a test cannot be undertaken at present because INTEGRAL-SPI does not have pinpointing capabilities to measure radiation from nebulas with high sensitivity, but the next-generation gamma-ray detectors (such as the Advanced Compton Telescopes) should be able to perform such measurements.
Another effect we have investigated is the overall contribution of PAHs to the $511 \mathrm{keV}$ line from the ISM, recalling that we now have high-quality spectra of the annihilation emission from the galactic center region measured by INTEGRAL-SPI (Jean et al. 2006). We attempted to relate the reaction rate we have calculated to the annihilation spectra that have been reduced from the INTEGRAL-SPI data. We applied the same method as in Jean et al. (2006), but instead of fitting the contribution of grains $\left(x_{g r}\right.$ in Eq. 3 of Jean et al. 2006) we fit the parameter $x_{P A H}=Y_{P A H}\left\langle f_{\text {elec }}\right\rangle$ to the measured spectrum. We obtained an upper-limit $x_{P A H}<3.0 \times 10^{-7}$. This upper-limit is in the range of expected values of $Y_{P A H}$ and $\left\langle f_{\text {elec }}\right\rangle$. However, the annihilation rate per unit density of $\mathrm{PAH}$ used for this fit is affected by an uncertainty of $47 \%$. Therefore, the formal upper-limit should be $x_{P A H}<4.6 \times 10^{-7}$. This defines a limit in the $Y_{P A H}-\left\langle f_{\text {elec }}\right\rangle$ space beyond which these parameters are not allowed, or else the effect would have been seen in the spectral analysis of the annihilation emission measured by SPI. Figure 4 shows the authorized domain $x_{P A H}=Y_{P A H} \times\left\langle f_{\text {elec }}\right\rangle<4.6 \times 10^{-7}$. For instance, if all PAHs are negatively charged, then their abundance in the Galactic bulge should be less than $1.3 \times 10^{-7}$. When all PAHs are neutral, their abundance should be less than 4.6 $\times 10^{-7}$.

This result is very consistent with other estimations, which are obtained from totally different approaches: using analysis of Infrared Space Observatory (ISO) observations of the PAH emission in the Galaxy, Wolfire et al. (2003) obtain a total PAH abundance of $6 \times 10^{-7}$; from their spectral analysis of $[\mathrm{SiPAH}]^{+}$complexes (the $6.2 \mu \mathrm{m}$ AIB), Joalland et al. (2009) infer a PAH-to-H abundance of $8 \times 10^{-7}$. We should note, however, that such measurements are not from the galactic bulge, where the presence and amounts of PAHs is unknown, so we cannot in all rigor compare the two results, despite their tantalizing closeness. Indeed, detections of PAH IR emission has been done from almost everywhere in the Galaxy (see earlier references plus Giard et al. 1988, 1989, and Giard et al. 1994), but not the bulge itself $(l<8 \mathrm{deg})$; in the bulge, however, emission features of PAH molecules have been detected in planetary nebulae (Perea-Calderón et al. 2009; Phillips \& Ramos-Larios 2009). 


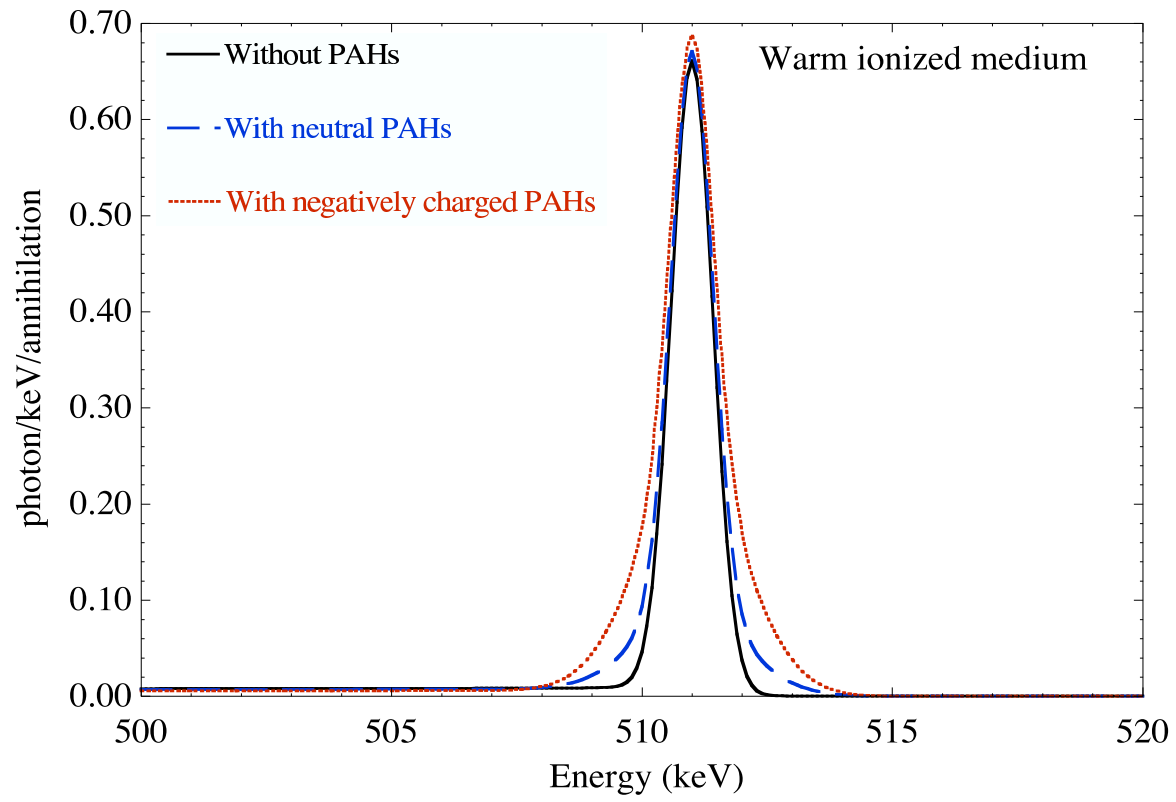

Figure 3. Spectral distribution of the annihilation emission in the warm ionized medium with and without PAH contribution, calculated assuming an abundance of PAHs of $Y_{P A H} \sim 10^{-6}$. The cases of neutral and negatively charged PAHs are presented separately.

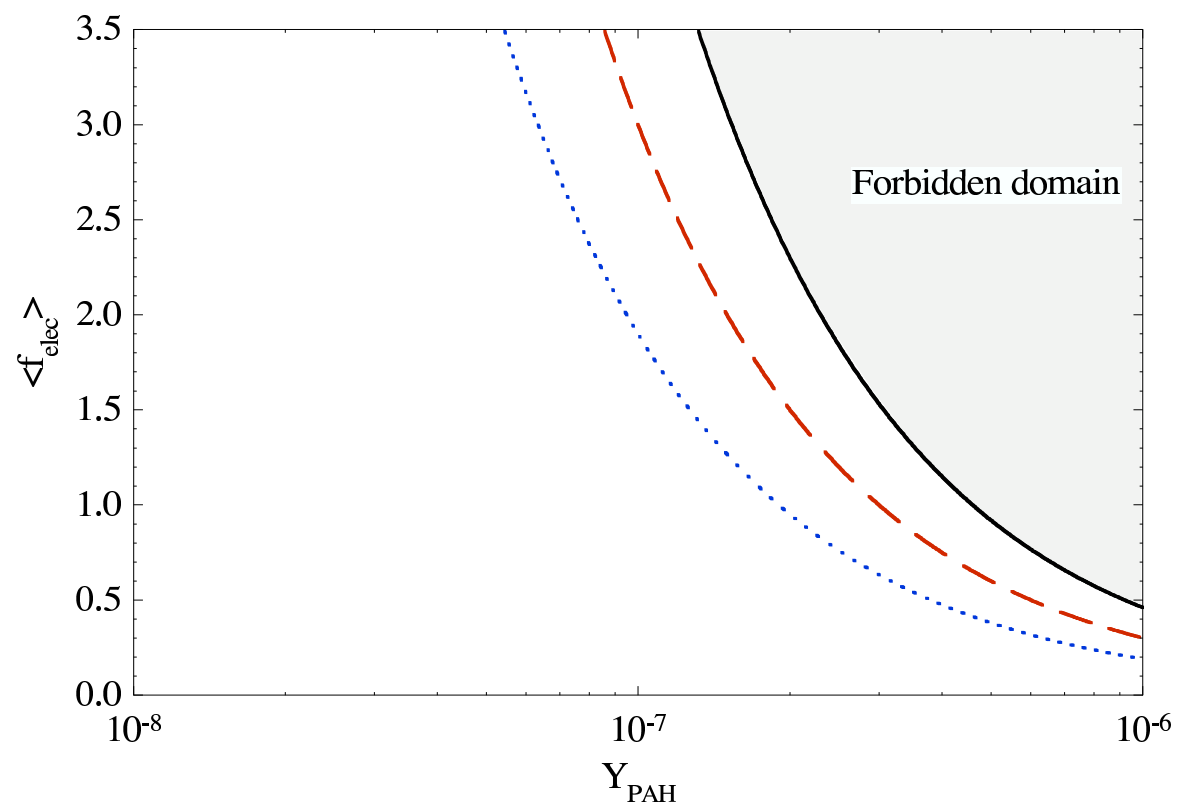

Figure 4. Domain of $Y_{P A H}$ and $\left\langle f_{\text {elec }}\right\rangle$ values authorized by the SPI data: $x_{P A H}<4.6 \times 10^{-7}$. The dashed and dotted lines correspond to $x_{P A H}=3.0 \times 10^{-7}$ and $x_{P A H}=1.9 \times 10^{-7}$, respectively.

\section{SUMMARY AND CONCLUSIONS}

The contribution of PAHs to the annihilation of positrons in the ISM had not been considered up till now. The simple reason for this is that PAHs have always been considered as the smallest type of dust grains, and since the cross section for positron annihilation on grains had been shown (Zurek 1985) to be geometrical (modulo electric and destruction effects), the contribution of the (extremely small) PAHs was dismissed as negligible (Guessoum. Jean, \& Gillard 2005). The error in that consideration was in overlooking the huge resonances in positron-PAH reactions (which, furthermore, did not occur for electrons), resonances that were vibrational in nature and led to increases in the annihilation cross sections by factors of up to $10^{7}$. This is what prompted us to investigate this effect in its potential astrophysical applications.

Our current knowledge of $\mathrm{e}^{+}-\mathrm{PAH}$ reactions is still very limited, both from experimental and theoretical perspectives. We have thus undertaken to estimate the rate of positron annihilation on PAH molecules in the ISM by a semi-empirical method. We have used all the information available to us from laboratory measurements of cross sections and from space IR analyses and estimates of abun- 
Annihilation of Positrons on PAHs in the ISM

dances and distributions of PAHs in the ISM. We have had to make some extrapolations, which we have shown to be uncertain but rather reasonable, from alkane data to $\mathrm{PAH}$ values and from low temperatures to ISM temperatures.

We have found that large molecules (with a total number of atoms of about 50) are most important for positron annihilation. We have also obtained a total rate of positron annihilation on PAH molecules, and we have found that if the total amount of PAH (relative to hydrogen) in the ISM is of the order of $10^{-6}$, then this process becomes second in importance in the warm neutral and the warm ionized phases, especially if the molecules are negatively charged. The issue of the charge states of the PAHs in various ISM conditions complicates the problem somewhat; indeed, depending on the temperature but particularly on the intensity of the UV field in the region, the PAHs may be neutral, positively, or negatively charged, and this would enhance or reduce their reaction rates with the positrons.

We have emphasized the large uncertainties inherent in this first attempt at tackling the problem, and we have pointed to the necessary future experimental, observational, and theoretical work for a substantial improvement of our understanding of this issue. In particular, we have called for experimental measurements of cross sections of positron annihilation on large PAH molecules. We also hope that future IR research, particularly with the Spitzer telescope, will help determine more precisely the specific PAHs which exist in space as well as their relative abundances in the ISM.

Finally, we have pointed to at least one future observational test, namely the measurement of the $511 \mathrm{keV}$ line from specific nebulas where PAHs may be particularly abundant and where physical conditions may have led to substantial negative charging of the molecules and hence to enhanced positron annihilation rates. As we explained, such observational tests cannot be undertaken with current gamma-ray instruments, but the next-generation gamma-ray detectors should be able to. We have also attempted to relate the reaction rate we have calculated to the annihilation spectra that have been reduced from the INTEGRAL-SPI data. We have shown that the spectral analysis may provide constraints on the abundance and the electric charge of PAH in the ISM. Using the semi-empirical model of annihilation of positrons with $\mathrm{PAH}$, the analysis of the spectrum of the annihilation emission from the galactic bulge measured by SPI tells us that the number-abundance of $\mathrm{PAH}$ relative to hydrogen should be less that $4.6 \times 10^{-7}$ if all PAHs are neutral and even lower if many of them are negatively charged.

\section{ACKNOWLEDGMENTS}

N. Guessoum acknowledges financial support from the American University of Sharjah through a research grant for this work and the Centre d'Etude Spatiale des Rayonnements for its hospitality and services during the conduct of this research. The authors thank Cliff Surko for pointing out the complications in estimating the rates at higher temperatures due to the change in the behavior of the resonant reactions above vibrational frequencies, Alain Kotz for reviewing and correcting our information on PAHs, Christine Joblin for useful discussions and pointers, and Paolo Pilleri for providing us with the PAH size distribution of Draine \& Lazarian (1998) in tabular form.

\section{REFERENCES}

Abergel, A., Verstraete, L., Joblin, C., Laureijs, R., \& Miville-Deschnes, M.-A. 2005, Sp. Sci. Rev., 119(1-4), 247271.

Allain, T. 1996, Photodestruction and Growth of Interstellar PAH. Ph. D. thesis, Berlin University.

Allamandola, L. J., Tielens, A. G. G. M., \& Barker, J. R. 1985, ApJ Lett., 290, 25-28.

Bakes, E. L. O. \& Tielens, A. G. G. M. 1994, ApJ, 427(2), 822-838.

Barnes, L. D., Gilbert, S. J., \& Surko, C. M. 2003, Phys. Rev. A, 67(3), 032706-1-11.

Barnes, L. D., Young, J. A., \& Surko, C. M. 2006, Phys. Rev. A, 74(1), 012706-1-10.

Cami, J., Tan, X., Biennier, L., \& Salama, F. 2005, In: Lis, D. C., Blake, G. A., and Herbst, E. (Eds.), Astrochemistry Throughout the Universe: Recent Successes and Current Challenges. IAU Symp. 231, Cambridge University Press, Cambridge, pp. 69-72.

Churazov, E., Sunyaev, R., Sazonov, S., Revnivtsev, M., \& Varshalovich, D. 2005, MNRAS, 357(4), 1377-1386.

Dartois, E. \& d'Hendecourt, L. 1997, A \& A, 323, 534-540.

Désert, F.-X., Boulanger, F., \& Puget, J. L. 1990, A \& A, 237(1), 215-236.

Draine B. T., Lazarian A., 1998, ApJ, 508, 157-179.

Draine, B. T. \& Sutin, B. 1987, ApJ, 320, 803-817.

Duley, W. W. \& Williams, D. A. 1981, MNRAS, 196, 269274.

Ehrenfreund, P. et al. 2002, ApJ Lett., 576(2), L117-L120.

Giard, M., Serra, G., Caux, E., Pajot, F. \& Lamarre, J. M. 1988, A \& A, 201(1), L1-L4.

Giard, M., Serra, G., Caux, E., Pajot, F. \& Lamarre, J. M. 1989, A \& A, 215(1), 92-100.

Giard, M., Lamarre, J. M., Pajot, F. \& Serra, G. 1994, A \& A, 286, 203-21.

Gilbert, A. M. et al. 2000, ApJ Lett., 533(1), L57-L60.

Gillett, F. C., Forrest, W. J., \& Merrill, K. M. 1973, ApJ, 183, 87-93.

Gribakin, G. F., 2000, Phys. Rev. A, 61(2), 022720-1-13.

Guessoum, N., Jean, P., \& Gillard, W. 2005, A \& A, 436(1), 171-185.

Guessoum, N., Jean, P., \& Prantzos, N. 2006, A \& A, 457(3), 753-762.

Guessoum, N., Ramaty, R., \& Lingenfelter, R. E. 1991, ApJ, 378, 170-180.

Haymes, R. C., Walraven, G. D., Meegan, C. A., Hall, R. D., Djuth, F. T., \& Shelton, D. H. 1975, ApJ, 201, 593602.

Harris, M. J. et al. 1998, ApJ Lett., 501, L55-L58.

Iwata, K., Greaves, R. G., Murphy, T. J., Tinkle, M. D., \& Surko, C. M. 1995, Phys. Rev. A, 51(1), 473-487.

Iwata, K. Greaves, R. G., \& Surko, C. M. 1996, Can. J. Phys., 74(7-8), 407-410.

Iwata, K., Greaves, R. G., \& Surko, C. M. 1997. $\gamma$-ray spectra from positron annihilation on atoms and molecules. Phys. Rev. A 55(5), 3586-3604.

Iwata, K., Gribakin, G. F., Greaves, R. G., Kurz, C., \& Surko, C. M. 2000, Phys. Rev. A, 61(2), 022719-1-17. 
Jean, P. et al. 2003, A \& A, 407, 55-58 .

Jean, P., Knödlseder, J., Gillard, W., Guessoum, N., Ferrière, K., Marcowith, A., Lonjou, V., \& Roques, J. P. 2006, A \& A, 445(2), 579-589.

Joalland, B., Simon, A., Marsden, C. J., Joblin, C. 2009, A \& A, 494, 969-976.

Johnson, W. N. III, Harnden, F. R. Jr., \& and Haymes, R. C. 1972, ApJ Lett., 172, L1-L7.

Johnson, W. N. III \& and Haymes, R. C. 1973, ApJ, 184, 103126.

Knödlseder, J. et al. 2005, A \& A, 441(2), 513-532.

Laricchia, G. \& Wilkin, C. 1997, Phys. Rev. Lett., 79(12), 2241-2244.

Laricchia, G. \& Wilkin, C. 1998, Nucl. Inst. and Meth. in Phys. Res. B 143(1-2), 135-139.

Leger, A. \& Puget, J. L. 1984, A \& A. Lett., 137(1), L5-L8. Lepp, S., Dalgarno, A., van Dishoeck, E. F., \& Black, J. H. 1988, ApJ, 329, 418-424.

Leventhal, M., MacCallum, C. J., \& Stang, P. D. 1978, ApJ Lett., 225, L11-L14.

Marler, J. P., Barnes, L. D.. Gilbert, S. J., Sullivan, J. P., Young, J. A., \& Surko, C. M. 2004, Nucl. Inst. and Meth. in Phys. Res. B 221, 84-92.

Milne, P. A., Kurfess, J. D., Kinzer, R. L., Leising, M. D., \& Dixon, D. D. 2000, In: McConnell, M. L. and Ryan, J. M. (Eds.), Proceedings of the fifth Compton Symposium, held in Portsmouth, NH, USA, September 1999. Melville, NY: American Institute of Physics (AIP), 510, 21.

Murphy, T. J. \& Surko, C. M. 1991, Phys. Rev. Lett., 67(21), 2954-2957.

Omont, A., 1986, A \& A, 164(1), 159-178.

Peeters, E., 2002. PAHs and dust in regions of massive star formation. Ph. D. thesis, Catholic University of Leuven.

Perea-Calderón, J. V., García-Herández, D. A., GarcíaLario, P., Szczerba, R. \& Bobrowsky, M. 2009, A \& A, 495(2), L5-L8.

Phillips, J. P. \& Ramos-Larios, G. 2009, MNRAS, 396(4), 1915-1928.

Pilleri, P., Herberth, D., Giesen, T. F., et al. 2009, MNRAS, 397, 1053-1060.

Purcell, W. R., Cheng, L.-X., Dixon, D. D., Kinzer, R. L., Kurfess, J. D., Leventhal, M., Saunders, M. A., Skibo, J. G., Smith, D. M., \& Tueller, J. 1997, ApJ, 491, 725-748.

Rapacioli, M. 2005, Des hydrocarbures aromatiques polycycliques à leurs agrégats: Études observationnelles et thóriques. PhD thesis, Université Toulouse III - Paul SABATIER.

Ruiterkamp, R., Cox, N. L. J., Spaans, M., Kaper, L., Foing, B. H., Salama, F., \& Ehrenfreund, P 2005, A \& A, 432(2), 515-529.

Salama, F., Galazutdinov, G. A., Krelowski, J., Allamandola, L. J., \& Musaev, F. A. 1999, ApJ, 526(1), 265-273.

Sullivan, J. P., Gilbert, S. J., Marler, J. P., Barnes, L. D., Buckman, S. J., \& Surko, C. M. 2002, Nucl. Inst. and Meth. in Phys. Res. B 192(1-2), 3-16.

Surko, C. M., Passner, A., Leventhal, M., \& Wysocki, F. J. 1988, Phys. Rev. Lett., 61(16), 1831-1834.

Surko, C. M., Greaves, R. G., Iwata1, K., \& Gilbert, S. J. 2000, Nucl. Inst. and Meth. in Phys. Res. B 171(1-2), $2-16$.

Surko, C. M., Gribakin, G. F., \& Buckman, S. J. 2005, Journal of Physics B, Atomic Molecular Physics 38(6),
R57-R126.

Surko, C. M. 2006, Nucl. Inst. and Meth. in Phys. Res. B 247(1), 1-4.

Tielens, A. G. G. M. 1990, In: Edited by F.-L. Navarria and P.G. Pelfer (Eds.), San Miniato Topical Seminar on Astrophysics and Particle Physics, Nuclear Physics B Proceedings Supplements, vol. 14, issue 1, pp. 13-17.

Weidenspointner, G. et al. 2006, A \& A, 450(3), 1013-1021. Weidenspointner et al. 2008, Nature, 451, L159-L162.

Weingartner, J. C. \& Draine, B. T. 2001a, ApJS, 134(2), 263-281.

Weingartner J. C. \& Draine, B. T. 2001b, ApJ, 563(2), 842-852.

Wolfire, M., McKee, C. F., Hollenbach, D. J., \& Tielens, A. G. G. M. 2003, ApJ, 587, 278-311.

Zurek, W. H. 1985, ApJ, 289, 603-608. 